\title{
Fuzzy determination of informative frequency band for bearing fault detection
}

\author{
Chuan Li $^{\mathrm{a}, \mathrm{b}, *}$, José Valente de Oliveira ${ }^{\mathrm{b}, \mathrm{c}}$, René-Vinicio Sanchez ${ }^{\mathrm{b}}$, Mariela Cerrada ${ }^{\mathrm{b}, \mathrm{d}}$, \\ Grover Zurita $^{\mathrm{b}}$ and Diego Cabrera ${ }^{\mathrm{b}}$ \\ ${ }^{a}$ National Research Base of Intelligent Manufacturing Service, Chongqing Technology and Business University, \\ Chongqing, China \\ ${ }^{\mathrm{b}}$ Department of Mechanical Engineering, Universidad Politécnica Salesiana, Cuenca, Ecuador \\ ${ }^{\mathrm{c}}$ CEOT, Universidade do Algarve, Faro, Portugal \\ ${ }^{\mathrm{d}}$ Control Systems Department-CEMISID, Universidad de Los Andes, Mérida, Venezuela
}

\begin{abstract}
Detecting early faults in rolling element bearings is a crucial measure for the health maintenance of rotating machinery. As faulty features of bearings are usually demodulated into a high-frequency band, determining the informative frequency band (IFB) from the vibratory signal is a challenging task for weak fault detection. Existing approaches for IFB determination often divide the frequency spectrum of the signal into even partitions, one of which is regarded as the IFB by an individual selector. This work proposes a fuzzy technique to select the IFB with improvements in two aspects. On the one hand, an IFB-specific fuzzy clustering method is developed to segment the frequency spectrum into meaningful sub-bands. Considering the shortcomings of the individual selectors, on the other hand, three commonly-used selectors are combined using a fuzzy comprehensive evaluation method to guide the clustering. Among all the meaningful sub-bands, the one with the minimum comprehensive cost is determined as the IFB. The bearing faults, if any, can be detected from the demodulated envelope spectrum of the IFB. The proposed fuzzy technique was evaluated using both simulated and experimental data, and then compared with the state-of-the-art peer method. The results indicate that the proposed fuzzy technique is capable of generating a better IFB, and is suitable for detecting bearing faults.
\end{abstract}

Keywords: Rolling element bearing, fuzzy clustering, fuzzy comprehensive evaluation, fault detection, envelope demodulation

\section{Introduction}

Healthy rolling element bearings are vital to the safe and effective operation of rotating machinery. Hence bearing fault detection is a crucial task in mechanical system maintenance [1]. To detect weak bearing faults as early as possible, different condition information has been reported with different applications [2-4]. Among these, vibration signal analysis has proven to be simple yet effective for bearing fault detection.

\footnotetext{
${ }^{*}$ Corresponding author. Chuan Li, National Research Base of Intelligent Manufacturing Service, Chongqing Technology and Business University, Chongqing 400067, China. Tel.: +86 236276 8469; Fax: +86 23 62769374; E-mail: E-mail: chuanli@21cn.com.
}

When a rolling element moves over a damaged surface, an impulsive force is produced that excites resonances in the mechanical system. The resonant responses may be modulated by the passage of the fault through the load zone or of the changing transmission path between the impact point and the measurement point [5]. In addition, the fault-induced impulsive features are often buried among background noise and interference, making bearing fault detection a challenge [6]. Vibration demodulation is an effective technique to recover weak impulsive features from contaminated and modulated raw signals [7]. The successive application of vibration demodulation to detect bearing faults comprises three main steps [8]: (1) determine the informative frequency band (IFB) around the resonance frequency of the 
system [9]; (2) bandpass filter the vibration signal using the IFB; and (3) generate the demodulated envelope spectrum to identify faulty characteristic frequency and its harmonics.

With a known pattern for the rotating machinery, one may employ the resonance frequency band of the system to bandpass filter the vibratory signal. If such prior knowledge is unavailable and there is a change in the severity of the defect, determining the IFB becomes the most challenging task when using vibration demodulation for bearing fault detection [10].

The determination of the IFB involves two main issues: the selector and the location of the informative band. Different statistical parameters have been employed as the selector to determine the IFB. Antoni and Randall [11] applied the kurtosis as a selector (statistical tool) for components taken from the spectrogram for each frequency bin. Bozchalooi and Liang [12] used a smoothness index for vibration signal denoising and fault detection. Gryllias and Antoniadis [13] proposed a peak energy criterion as the selector for bearing resonance bands. Obuchowski et al. [9] employed a set of statistics, namely Jarque-Bera, Kolmogorov-Smirnov, Cramervon Mises, Anderson-Darling, quantile-quantile plot and local maxima for IFB selection. According to the literature, kurtosis is sensitive to the impulsive feature included in the IFB, and hence widely used for bearing fault diagnosis. However, the kurtosis is also sensitive to spiking noises, and therefore prone to the influence of outliers. The smoothness index, though immune to outliers, is weak for discriminating signals of smaller value. The selectors based on statistical moments are more sensitive to single, incidental impulses. As for the empirical cumulative distribution function, the quantile-quantile plot and the local maxima, further processing methods are required in some cases. Considering the fact that a mono-selector is not versatile for all cases of bearing fault detection, Li et al. [14] suggested an entropy-weighted method using different selectors to guide bearing fault diagnosis. To our knowledge, these selectors all belong in the "hard" category and do not take into account the fuzzy nature of the attributes.

To determine the location of the IFB band, frequency, time-frequency, and nonlinear frames have been reported to cope with machinery vibratory signals. The most direct approach for determining the IFB is the frequency domain. Antoni [15] proposed a dyadic signal decomposition scheme incorporating kurtosis (kurtogram) or negentropy (inforgram).
Barszcz and Jabłoński [16] proposed a protrugram using the kurtosis of the envelope spectrum amplitudes of the demodulated signal as an extension of the kurtogram. For dyadic or similar signal decomposition, the frequency band is evenly divided into sub-bands to determine which sub-band is the best for the informative band. In the time-frequency plane, He et al. [17] determined the optimal bandpass filter using wavelets. Li and Liang [18] developed a generalized synchrosqueezing transform to perform a time-frequency analysis of the vibratory signals [19]. Moreover, in the nonlinear frame, Flandrin et al. [20] suggested empirical mode decomposition (EMD) as a filter bank for signal processing. Using a mathematical morphology technique, Li and Liang [21] reported a multi-scale autocorrelation approach incorporating mathematical morphological (MM) wavelet slices for the diagnosis of rolling element bearing faults. Optimal scale band demodulation of the impulsive feature has also been proposed for bearing defect diagnosis [22]. It should be noted that under the time-frequency frame, the optimized bandpass filter can be interpreted as a counterpart of the IFB. However, under a nonlinear frame such as EMD and MM, the optimal scale for signal denoising is not directly correlated to the IFB, but can be regarded as the mapping of the IFB in a nonlinear dimension. No matter which frame is exploited, the most popular methods for locating the informative band are based on the mean segmentation of the whole band (or scale). There are three natures of the conventional mean segmentation technique. First, mean segmentation lacks physical meaning due to its direct grouping. Second, the commonly-used dyadic or level-by-level decomposition exhibits coarse precision in determining the band edges. Third, these methods are a form of "hard" splitting that fail to take into account the fuzzy nature of the segments. As a pilot attempt, Hou et al. [23] proposed a clusteringbased segmentation approach for bearing vibratory signal demodulation. The internal homogeneity of the vibration data was taken into account to guide the frequency band clustering. Although data homogeneity is a good selector in normal cases, it is insufficient for fault diagnosis as the impulsive nature is ignored during clustering.

Inspired by the fact that real information is generally fuzzy instead of "hard" for different cases, this work proposes a fuzzy technique to improve the IFB determination performance in two aspects. First, an IFB specific fuzzy clustering method is developed to divide the frequency spectrum into meaningful, unequal sub-bands [24]. Second, three 
commonly-used criteria are combined using a fuzzy comprehensive evaluation method to guide the frequency band segmentation [25]. Among all the meaningful sub-bands, the one with the minimum comprehensive cost is determined as the IFB. Application of the above mentioned fuzzy techniques is evaluated using both simulated and experimental data. The proposed fuzzy technique replaces the classical "hard" approach to determine the IFB.

The rest of the paper is structured as follows. The domain-specific fuzzy clustering algorithm and fuzzy comprehensive evaluation of selectors are proposed in Section 2. The addressed fuzzy technique is applied to the bearing fault detection in Section 3. A simulation case is illustrated in Section 4 and three experiments with real data are shown in Section 5 to evaluate the proposed technique. Conclusions are given in Section 6.

\section{Fuzzy determination of the IFB}

\subsection{Fuzzy clustering of the frequency spectrum}

For a vibratory signal $x(t)$ collected from a rolling element bearing, its frequency spectrum $X(f)$ is given by

$$
X(f)=\mathscr{F}(x(t))=\int_{-\infty}^{+\infty} x(t) \mathrm{e}^{-2 \pi \mathrm{j} f t} \mathrm{~d} t,
$$

where $\mathscr{F}($.$) is the Fourier transform function. The$ frequency spectrum $X(f)\left(f=f_{1}, f_{2}, \ldots, f_{N}\right)$ is a finite set of $N$ spectral samples. A frequency band is a subset of $X(f)$ with consecutive frequency points

$$
B(a, b)=\left\{X\left(f_{a}\right), X\left(f_{a+1}\right), \ldots, X\left(f_{b}\right)\right\} .
$$

In this way, the $c$-segmentation of the frequency spectrum is a partition of $X(f)$ in $c$ non-overlapping frequency bands, i.e.,

$$
B_{X}^{c}=\left\{B_{i}\left(a_{i}, b_{i}\right) \mid 1 \leq c\right\},
$$

where $a_{1}=f_{1}, b_{c}=f_{N}$, and $a_{i}=b_{i-1}+1$.

As stated earlier, classical IFB determination techniques usually employ some time-frequency decomposition framework such as a short-time Fourier transform and wavelet transform for grouping the spectrum into mean partitions. Clustering is an effective tool to find internally homogeneous segments from a given series where consecutive data points are grouped in terms of their similarity. As the fuzzy C-means (FCM) generalizes disjoint cluster- ing, this work develops a variant of the classical FCM clustering for generating more meaningful, unequal sub-bands. The cost function of the FCM can be expressed as [26]

$$
J(\mu)=\sum_{i=1}^{N} \sum_{j=1}^{c} \mu_{i j}^{m} d_{i j}^{2},
$$

where $\mu_{i j}\left(0 \leq \mu_{i j} \leq 1\right)$ is the membership degree of the frequency point $X\left(f_{i}\right)$ in cluster $c_{j}, d_{i j}=$ $\left\|X\left(f_{j}\right)-e_{j}\right\|$ is the distance or dissimilarity between the frequency point $X\left(f_{i}\right)$ and the $j$ th cluster center $e_{j}$, and $m$ is a real number greater than 1 [27].

The distance $d_{i j}=\left\|X\left(f_{i}\right)-e_{j}\right\|$ for the FCM is a geometric definition of the dissimilarity. However, the impulsiveness resulting from the bearing fault should be reflected by other statistical criteria such as kurtosis. Hence the classical FCM in frequency spectrum clustering for bearing fault detection lacks the physical meaning of impulsiveness. Hence we develop a FCM variant for this problem that takes into account two aspects. First, the frequency spectrum $X(f)$ is a series, which restricts the non-null membership degrees of a frequency point $X\left(f_{i}\right)$ to only consecutive clusters, i.e.,

$$
\mu_{i j}=\left\{\begin{array}{ll}
\mu_{i j} & ; f_{i} \in\left[c_{j-1}, c_{j+1}\right] \\
0 & ; \text { Otherwise }
\end{array}, \sum \mu_{i j}=1 .\right.
$$

Second, our goal for the frequency spectrum clustering is to find the IFB whose selectors are not identical to the classical distance $d_{i j}$. Nevertheless, the IFB is still an internally homogeneous sub-band. Hence we propose the following dissimilarity index to replace the usual FCM distance $\left\|X\left(f_{j}\right)-e_{j}\right\|$ by:

$$
d_{i j}=N\left(H_{i j}\right)+N\left(S_{i j}\right),
$$

where $\mathcal{N}($.) stands for a normalized function that assigns the same importance to its arguments $\left(S_{i j}\right.$ and $\left.H_{i j}\right), S_{i j}$ being the selector cost which will be highlighted in the next subsection, and $H_{i j}$ denoting the homogeneity between the frequency point $X\left(f_{i}\right)$ and the $j$ th cluster center $e_{j}$, as given by

$$
H_{i j}=\sqrt{\frac{\sum_{k=a_{i}}^{e_{j}}\left(X\left(f_{k}\right)-\overline{X\left(f_{a i}, f_{e j}\right)}\right)^{2}}{e_{j}-a_{i}} / \overline{X\left(f_{a i}, f_{e j}\right)}},
$$

where $\overline{X\left(f_{a i}, f_{e j}\right)}$ is the mean of the frequency band between $f_{a i}$ and $e_{j}$. 
With these two improvements, one can employ the expectation-maximization algorithm to update $e_{j}$ and $\mu_{i j}$ towards the optimal solutions (i.e., $e_{j o p t}$ and $\left.\mu_{i j \mathrm{opt}}\right)$ of the segments [28]. After obtaining $e_{j \mathrm{opt}}$ and $\mu_{i j o p t}$, each frequency point can be grouped into one of the $c$ segments using the following equation:

$$
X\left(f_{i}\right) \in \begin{cases}B\left(a_{j}, b_{j}\right) & ; \mu_{k j \mathrm{opt}}>\mu_{(k, j+1) \mathrm{opt}} \\ B\left(a_{j+1}, b_{j+1}\right) & \text { for all } k \in\left[a_{j}, i\right] \\ \text { otherwise }\end{cases}
$$

In this way, the frequency spectrum is divided as the sum of $c$ sub-bands, i.e.,

$$
\left.X(f)\right|_{f=f_{1}, \ldots, f_{N}}=B\left(a_{1}, b_{1}\right) \cup \ldots \cup B\left(a_{c}, b_{c}\right) .
$$

\subsection{Fuzzy comprehensive evaluation of IFB selectors}

As introduced in the previous subsection, the selector cost $S_{i j}$ should be calculated for the application in the proposed clustering method. It is well known that the vibratory signal for a rolling element bearing with a defective inner race, outer race or ball exhibits cyclical impulsive features. As the impulses are usually modulated in the high-frequency resonance band, the one with the most evident impulsive characteristics can be regarded as the IFB. By analyzing the statistical indexes of the bearing vibratory signal, one can characterize its cyclical impulsive component. Hence in fault detection the effective statistical indexes have been employed as "selectors" [9], the commonly used ones among them being the kurtosis, smooth index and crest factor.

For the $j$ th frequency band $B\left(a_{j}, b_{j}\right)$ computed from the bearing vibratory signal $x(t)$, the corresponding temporal component is expressed as

$$
x_{j}(t)=\mathscr{F}^{-1}\left[B\left(a_{j}, b_{j}\right)\right],
$$

where $\mathscr{F}^{-1}$ denotes the inverse Fourier transform. The kurtosis of $x_{j}(t)$ is given by

$$
K\left(x_{j}(t)\right)=\frac{E\left\{x_{j}(t)-E\left\{x_{j}(t)\right\}^{4}\right\}}{E\left\{\left(x_{j}(t)-E\left\{x_{j}(t)\right\}\right)^{2}\right\}^{2}},
$$

where $E\{$.$\} represents the mathematical expectation.$ Generally, stronger impulses generate greater kurtosis values. Hence $1 / K\left(x_{j}(t)\right)$ can be employed as the selector cost for kurtosis. As introduced in Ref. [12], kurtosis is sensitive to all impulses, including spiking outliers. If spiking noises occur in the non-resonance band then the kurtosis selector may wrongly identify the non-resonance band as the IFB.

Compared with the kurtosis, the smoothness index is insensitive to the outlier. The definition of the smooth index is [12]

$$
H\left(x_{j}(t)\right)=\frac{G M\left(x_{j}(t)\right)}{A M\left(x_{j}(t)\right)},
$$

where $A M($.$) and G M($.$) represent the arithmetic$ mean and the geometric mean, respectively, which are given by

$$
\begin{aligned}
A M\left(x_{j}(t)\right) & =\frac{1}{M} \sum_{t=1}^{M} x_{j}(t), G M\left(x_{j}(t)\right) \\
& =\left(\prod_{t=1}^{M} x_{j}(t)\right)^{\frac{1}{M}},
\end{aligned}
$$

where $M$ is the length of the discrete time $t$. Usually, a smaller smoothness index results from a greater impulsive component. Hence the selector cost of the smoothness index is $H\left(x_{j}(t)\right)$ itself. Although the smoothness index is immune to the outlier, Gryllias and Antoniadis [13] point out that the smoothness index is actually insensitive to smaller values.

The crest factor acting as an IFB selector is similar to kurtosis. The greater the impulsive component, the bigger the crest factor will be. For the temporal signal $x_{j}(t)$ corresponding to the $j$ th frequency band, its crest factor is expressed as

$$
C\left(x_{j}(t)\right)=\frac{\max \left(x_{j}(t)\right)-A M\left(x_{j}(t)\right)}{R M S\left(x_{j}(t)\right)},
$$

where $R M S($.$) is the root mean square function given$ by

$$
R M S\left(x_{j}(t)\right)=\sqrt{\frac{1}{M} \sum_{t=1}^{M} x_{j}^{2}(t) .}
$$

Similar to kurtosis, the selector cost of the crest factor can be express as $1 / C\left(x_{j}(t)\right)$. Compared with kurtosis, the crest factor is less sensitive to outliers, but also less sensitive to the cyclical impulses.

Based on the aforementioned analysis, it is clear that any one of the above selectors is insufficient to deal with different complicated bearing vibratory signals, although individually they can be effective for the selection of the IFB in certain cases. On the one hand, real information is generally fuzzy instead of "hard" for different cases. On the other hand, the fuzzy comprehensive evaluation is a powerful tool for 
combining different selectors in an integrated fashion. Hence in this work the fuzzy comprehensive evaluation is applied to combine different selectors for the selection of the IFB, i.e. [29],

$$
A_{j}=F\left(I_{n}\left(x_{j}(t)\right)\right)
$$

where $F($.$) is the fuzzy comprehensive evaluation$ model, and $I_{n}$ is the $n$th mono-selector cost. In this work, we have

$$
\begin{aligned}
& I_{1}\left(x_{j}(t)\right)=\frac{1}{K\left(x_{j}(t)\right)}, I_{2}\left(x_{j}(t)\right)=H\left(x_{j}(t)\right), \text { and } \\
& I_{3}\left(x_{j}(t)\right)=\frac{1}{C\left(x_{i}(t)\right)} .
\end{aligned}
$$

For each frequency point $X\left(f_{i}\right)$ in cluster $c_{j}$, the temporal waveform correlated to its band can be denoted as $x_{i j}(t)$. Putting all $x_{i j}(t)$ together, one has the following series

$$
\begin{aligned}
\mathbf{x}=\{ & x_{11}(t), x_{21}(t), \ldots, x_{N 1}(t), x_{12}(t), \ldots, x_{N 2}(t) \\
& \left., \ldots, x_{1 c}(t), x_{2 c}(t), \ldots x_{N c}(t)\right\}
\end{aligned}
$$

Normalizing all the mono-selector cost values to $[0,1]$ yields the following vector

$$
L I_{n}(\mathbf{x})=\frac{I_{n}\left(x_{i j}(t)\right)-\min _{k=1}^{N c}\left(I_{n}\left(x_{i j}(t)\right)\right)}{\max _{k=1}^{N c l}\left(I_{n}\left(x_{i j}(t)\right)\right)-\min _{k=1}^{N c l}\left(I_{n}\left(x_{i j}(t)\right)\right)} .
$$

For the $n$th selector, the mean function $\overline{L I}_{n}$ and the standard deviation $\sigma_{n}$ of its normalized vector are respectively expressed as

$$
\begin{aligned}
\overline{L I}_{n} & =\frac{1}{N c} \sum_{k=1}^{N c} L I_{n}(\mathrm{x}), \\
\sigma_{n} & =\frac{1}{N c-1} \sqrt{\sum_{k=1}^{N c}\left(L I_{n}-\overline{L I}_{n}\right)^{2} .}
\end{aligned}
$$

Furthermore, one can calculate the total mean $\overline{L L}_{0}$ and total standard deviation $\sigma_{0}$ for all the selector costs as

$$
\overline{L I}_{0}=\frac{1}{n} \sum_{n} C \overline{L I}_{n}, \sigma_{0}=\frac{1}{2} \sqrt{\sum_{n}\left(\overline{L I}_{n}-\overline{L I}_{0}\right)^{2}} .
$$

By employing a triangular membership function, the fuzziness of the $n$th selector cost can be calculated as [30]

$$
\begin{aligned}
\tilde{I}_{n} & =\left\{p_{n 1}, p_{n 2}, p_{n 3}\right\} \\
& =\left\{\overline{L I}_{n}-2 \sigma_{n}, \overline{L I}_{n}, \overline{L I}_{n}+2 \sigma_{n}\right\} .
\end{aligned}
$$

Similarly, the fuzziness of the total selector cost can be expressed as

$$
\begin{aligned}
\tilde{I}_{0} & =\left\{p_{01}, p_{02}, p_{03}\right\} \\
& =\left\{\overline{L I}_{0}-2 \sigma_{0}, \overline{L I}_{0}, \overline{L I}_{0}+2 \sigma_{0}\right\} .
\end{aligned}
$$

The nearness between the $n$th cost fuzziness and the total cost fuzziness is therefore calculated as

$$
T_{n}=\frac{1}{1+\left|\frac{p_{n 1}+4 p_{n 2}+p_{n 3}-p_{01}-4 p_{02}-p_{03}}{6}\right|} .
$$

In real applications, a large $T_{n}$ means that the $n$th cost fuzziness is nearer to the total cost fuzziness, and vice visa. Hence the weight for the $n$th selector should be proportional to its nearness. Based on this observation, combining Equations (16-19) yields

$$
S_{i j}=\frac{T_{n}}{\sum_{n} T_{n}} L I_{n}\left(x_{i j}(t)\right) .
$$

Plugging Equation (25) into Equation (6) can generate the whole dissimilarity index $d_{i j}$ to guide the clustering process of the frequency spectrum.

\section{Application to the bearing fault detection}

\subsection{Bearing fault detection using the IFB}

According to the aforementioned fuzzy technique, a required parameter for the clustering procedure is the number of clusters $c$. Although in general it would be difficult to find an optimal number $c$ for different cases, we can follow the idea of the kurtogram to segment the frequency spectrum into different bands $B_{j c}$ for $(c=1,2,3, \ldots P)$, as shown in Fig. 1. Among the different bands, the one corresponding to the minimum selector cost $(\min S)$ is the IFB $B_{\text {opt }}$, whose corresponding $c$ is the optimal number of segments $c_{\text {opt }}$.

After determining of the IFB $X\left(f_{\text {opt }}\right)=B_{\text {opt }}$ $\left(a_{\text {opt }}, b_{\text {opt }}\right)$, one can perform the envelope demodulation as

$$
\left.v(t)=\bmod \left(H^{-1}\left(X\left(f_{\text {opt }}\right)\right)\right)\right) .
$$

where $v(t)$ is the demodulated envelope from the IFB, $\bmod ($.) stands for modulo operation, and $H$ (.) represents the Hilbert transform.

Applying the Fourier transform to the demodulated envelop $v(t)$, the obtained envelope spectrum $v(f)$ 


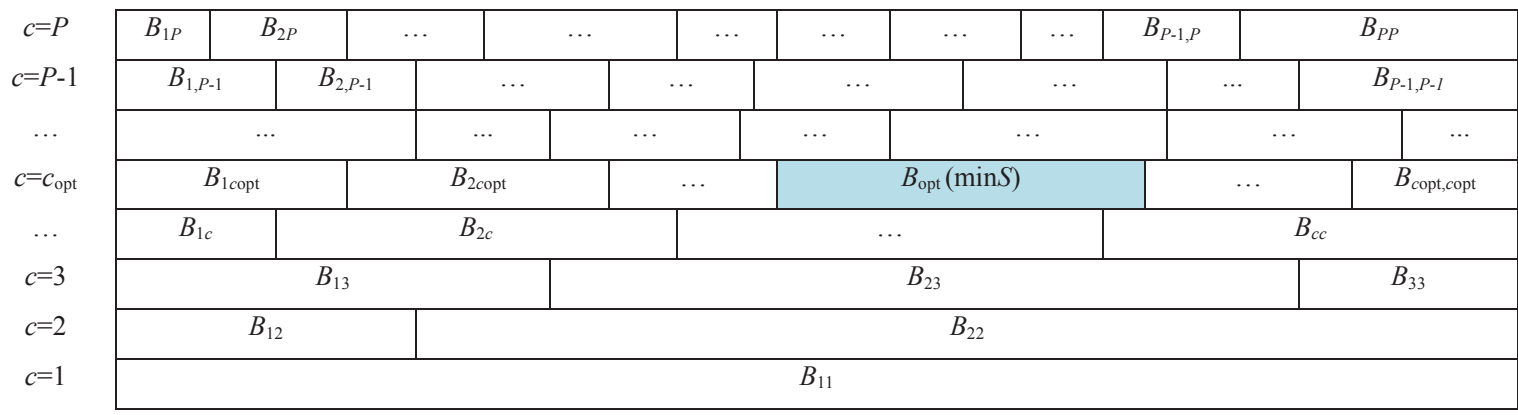

Fig. 1. Determination of the IFB using different $\mathrm{c}$ values.

can be used to detect the possible bearing fault. For a defective bearing, the ball pass frequency of the outer ring (BPFO) and the ball pass frequency of inner ring (BPFI) can be calculated as [31]

$$
\begin{aligned}
\operatorname{BPFO}\left(f_{c}\right) & =\frac{w}{2}\left(1-\frac{d}{D} \cos \alpha\right) f_{r}, \text { and } \\
\operatorname{BPFI}\left(f_{c}\right) & =\frac{u}{2}\left(1+\frac{d}{D} \cos \alpha\right) f_{r},
\end{aligned}
$$

where $w$ is the number of rolling elements, $d$ the diameter of the rolling element, $D$ the pitch diameter, $\alpha$ the contact angle, $f_{c}$ the characteristic frequency, and $f_{r}$ the rotating frequency. If the faulty characteristic frequency (e.g., BPFO, BPFI) and its harmonics can be identified from $V(f)$, one can accordingly detect the existence of a bearing fault.

\subsection{Overview of the present technique for the bearing fault detection}

Based on the aforementioned explanations, Fig. 2 illustrates the flowchart for the present technique used for bearing fault detection, which is also summarized below.

Step 1. Collect vibratory signal $x(t)$ from the rolling element bearing to be monitored;

Step 2. Calculate its Fourier spectrum $X(t)$ using Equation (1);

Step 3. Perform specific fuzzy clustering (as detailed in section 2) of the frequency spectrum with $c=1,2,3 \ldots, P$, and the dissimilarity depicted by Equation (6);

Step 4. From all the clustered sub-bands, determine the IFB $B_{\text {opt }}$ with the minimum selector cost $(\min S)$ as described in Fig. 1;

Step 5. Calculate the optimal demodulated envelope $v(t)$ from the obtained IFB using Equation (26);

Step 6. Calculate the envelope spectrum $V(f)$ by applying the Fourier transform to $v(t)$; and

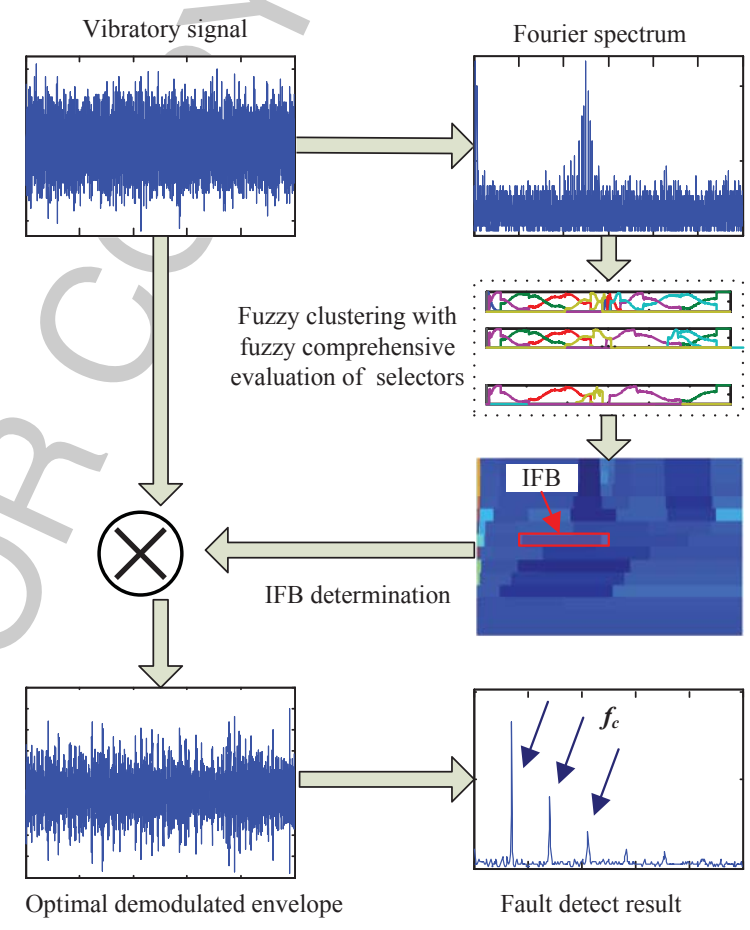

Fig. 2. Procedure of the present fuzzy technique for the bearing fault detection.

Step 7. Detect the bearing fault by identifying $f_{c}$ Equation (27) and its harmonics in $V(f)$, if any. End.

\section{Evaluation using simulated data}

For a defective rolling element bearing, its vibration measurement $x(t)$ can be simulated by [17]

$$
x(t)=G(t) \sum_{n} u\left(t-q / f_{c}\right)+\theta(t)+\delta(t),
$$

where $\delta(t)$ is the noise component, $\theta(t)$ the interference harmonic component, $G(t)$ the amplitude of the 

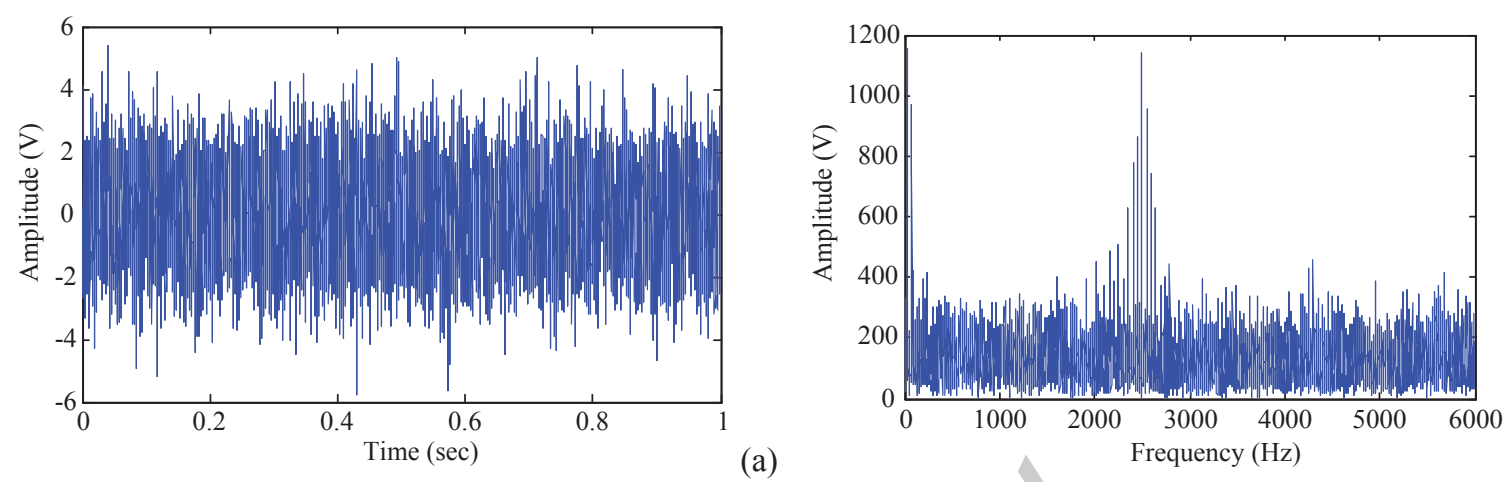

(b)
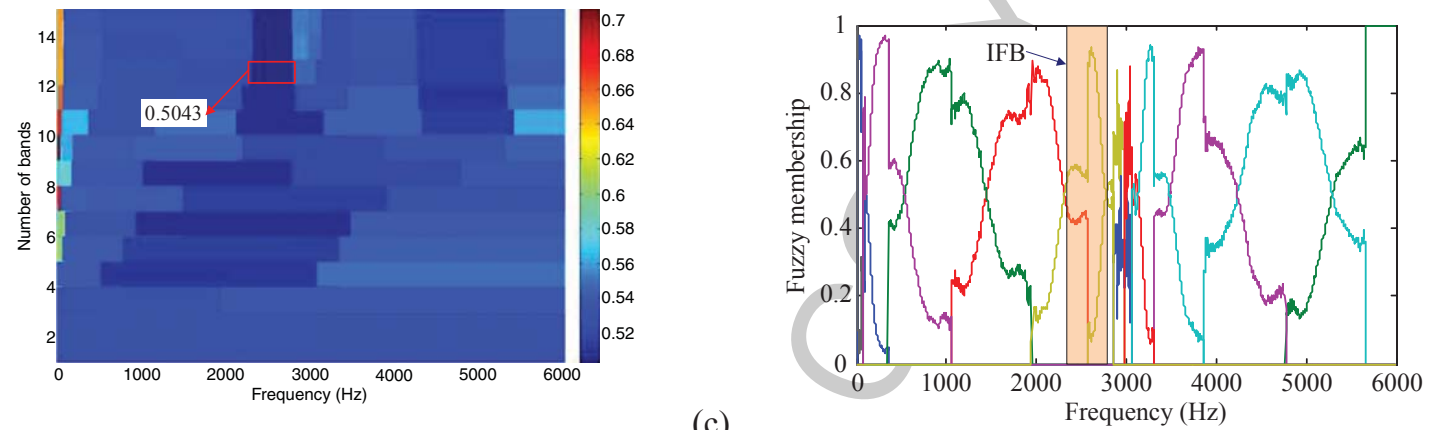

(d)

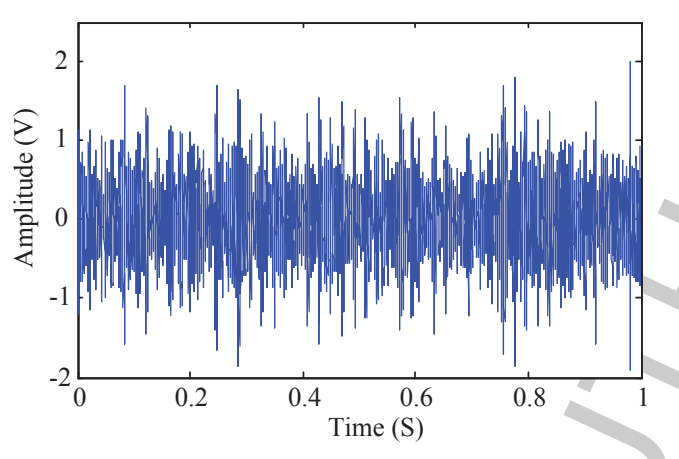

(c)

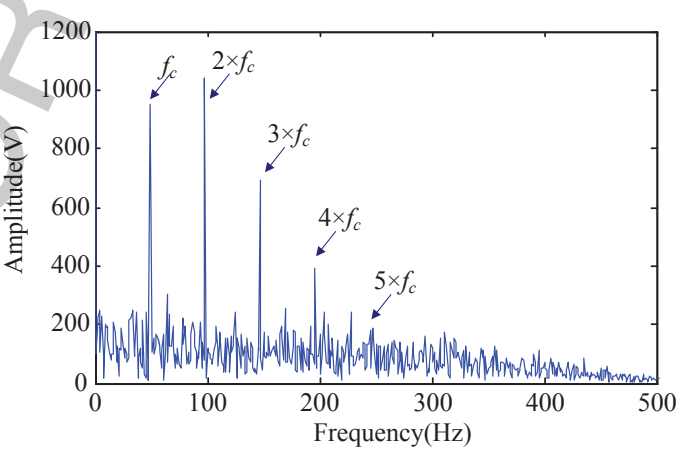

Fig. 3. Evaluate the present fuzzy technique using the simulated bearing data: (a) temporal waveform; (b) Fourier representation; (c) clustering results; (d) membership curves when $c_{\mathrm{opt}}=13$, (e) optimal demodulated envelope $v(t)$ from the IBF, and (f) its Fourier representation $V(f)$.

fb-kurt.2 - Kmax=0.5@level 2.5, Bw=1000Hz, $\mathrm{f}_{\mathrm{c}}=2500 \mathrm{~Hz}$
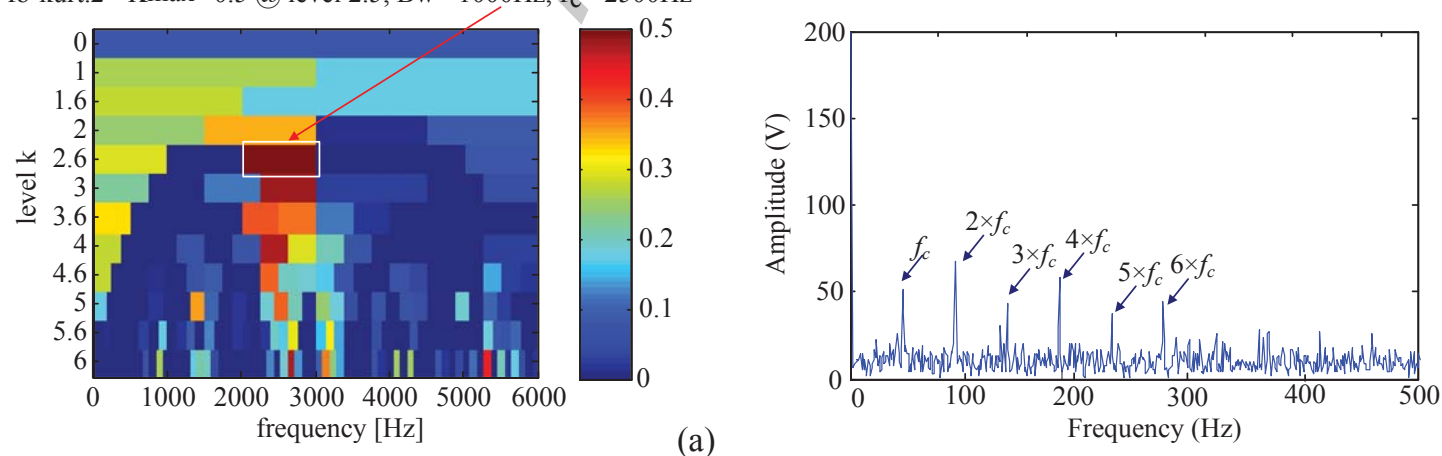

Fig. 4. Simulated fault detection using the kurtogram: (a) result of the kurtogram; and (b) envelope demodulation result from the IFB determined by the kurtogram. 


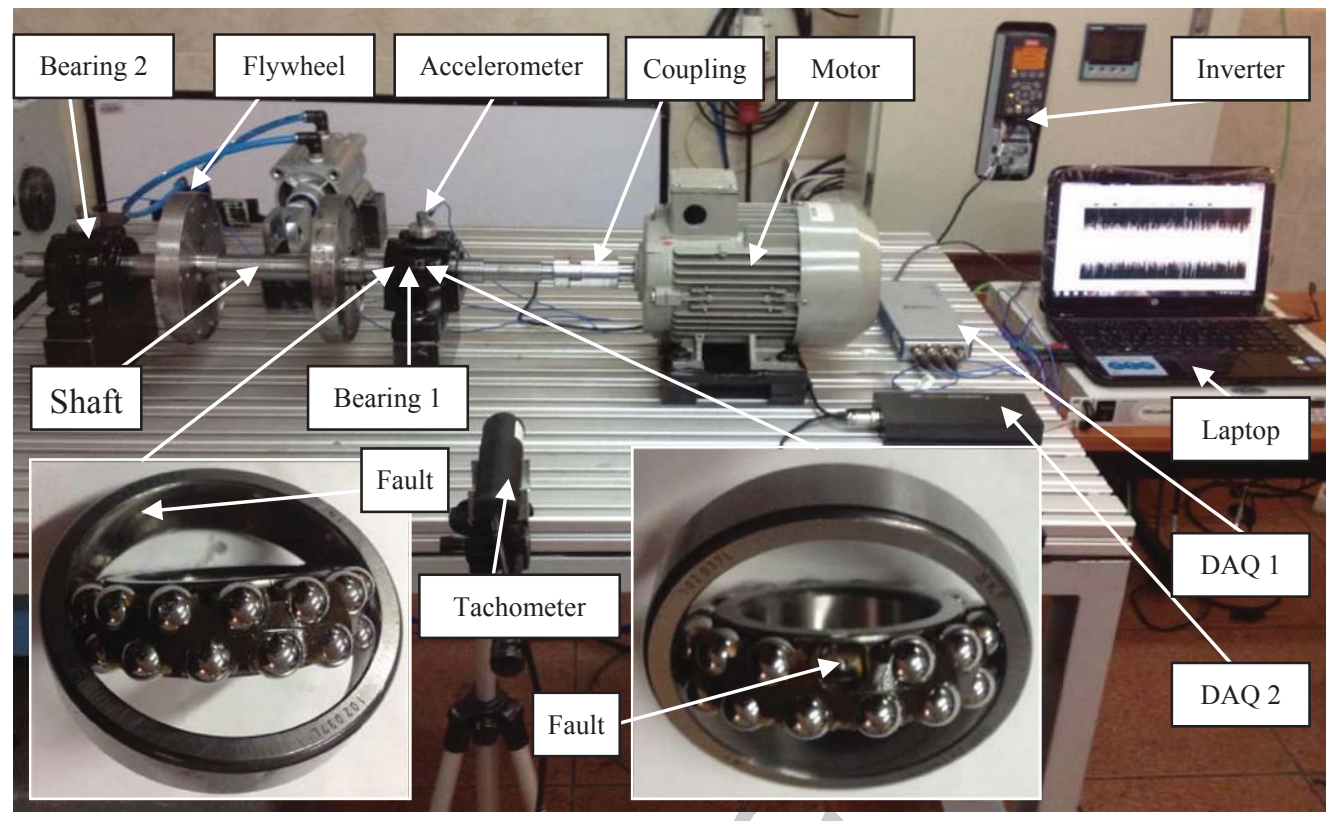

Fig. 5. Experimental setup for the bearing fault detection.

impulsive component, $q$ the number of impulses, $f_{c}$ the faulty characteristic frequency, and $u(t)$ the periodic impulse response function given by

$$
u(t)= \begin{cases}e^{-d_{b} t} \sin \left(2 \pi f_{0} t\right) & ; t>0 \\ 0 & ; t \leq 0\end{cases}
$$

where $d_{b}$ represents the band width, and $f_{0}$ denotes the central frequency of the informative (resonance) frequency band.

Following the vibratory signal model formulated by Equations (28) and (29), a simulated faulty bearing is considered with following parameters: $G(t)=[2.2,2.6] \mathrm{V}, d_{b}=650 \mathrm{~Hz}, f_{c}=49 \mathrm{~Hz}$, $f_{0}=2500 \mathrm{~Hz}, \theta(t)$ is the sum of the two harmonic components, i.e., $0.2 \sin (163 \pi t)$ and $0.3 \cos (61 \pi t)$, and $\delta(t)$ is Gaussian white noise with signal-to-noise ratio (SNR) $-10 \mathrm{~dB}$. Letting sampling frequency $f_{s}=$ $12000 \mathrm{~Hz}$ and the simulation duration $1 \mathrm{sec}$, one can obtain the temporal waveform of the simulated signal plotted in Fig. 3(a), and its Fourier spectrum as shown in Fig. 3(b).

The proposed fuzzy determination technique is then applied for the Fourier spectrum clustering with $c=1,2, \ldots, 15$. The clustering results for the Fourier spectrum are displayed in Fig. 3(c) where the IFB is automatically identified as $B_{\mathrm{opt}}=$ [2311, 2783] Hz at the 13th clustering ( $\left.c_{\mathrm{opt}}=13\right)$. Notice that in this figure dark blue areas correspond to higher values of membership degrees. To further illustrate the clustering process, the obtained membership curves at $c_{\mathrm{opt}}=13$ are shown in Fig. 3(d). From the IFB, the optimal demodulated envelope $v(t)$ of the IFB is calculated and shown in Fig. 3(e). Its frequency representation $V(f)$ is plotted in Fig. 3(f), where the faulty characteristic frequency $\left(f_{c}=\right.$ $49 \mathrm{~Hz}$ ) and its harmonics can be clearly identified.

For comparison, we apply the kurtogram to determine the IFB for the same simulated signal. The kurtogram of the simulated signal $x(t)$ is plotted in Fig. 4(a) where one can identify the IFB as [2000, $3000] \mathrm{Hz}$ corresponding to the maximum kurtosis. The spectral result obtained by envelope demodulation is shown in Fig. 4(b). A comparison of Figs. 3(f) and 4(b) reveals that the SNR shown in Fig. 3(f) is better than that in Fig. 4(b). This is due to a more precise estimation of the IFB by the present fuzzy technique. As modeled by Equations (28) and (29), the given resonance band is $[2175,2825] \mathrm{Hz}$. The present fuzzy technique with identified [2311, 2783] $\mathrm{Hz}$ accounts for a $32.57 \%$ difference from the resonance band, due to noise contamination. In contrast, the kurtogram with identified [2000, 3000] Hz produces a $42.86 \%$ error with the given resonance band, which introduces more noise to the bearing fault diagnosis. The comparison result indicates that the present fuzzy technique is more effective for the IFB determination in bearing fault detection. 

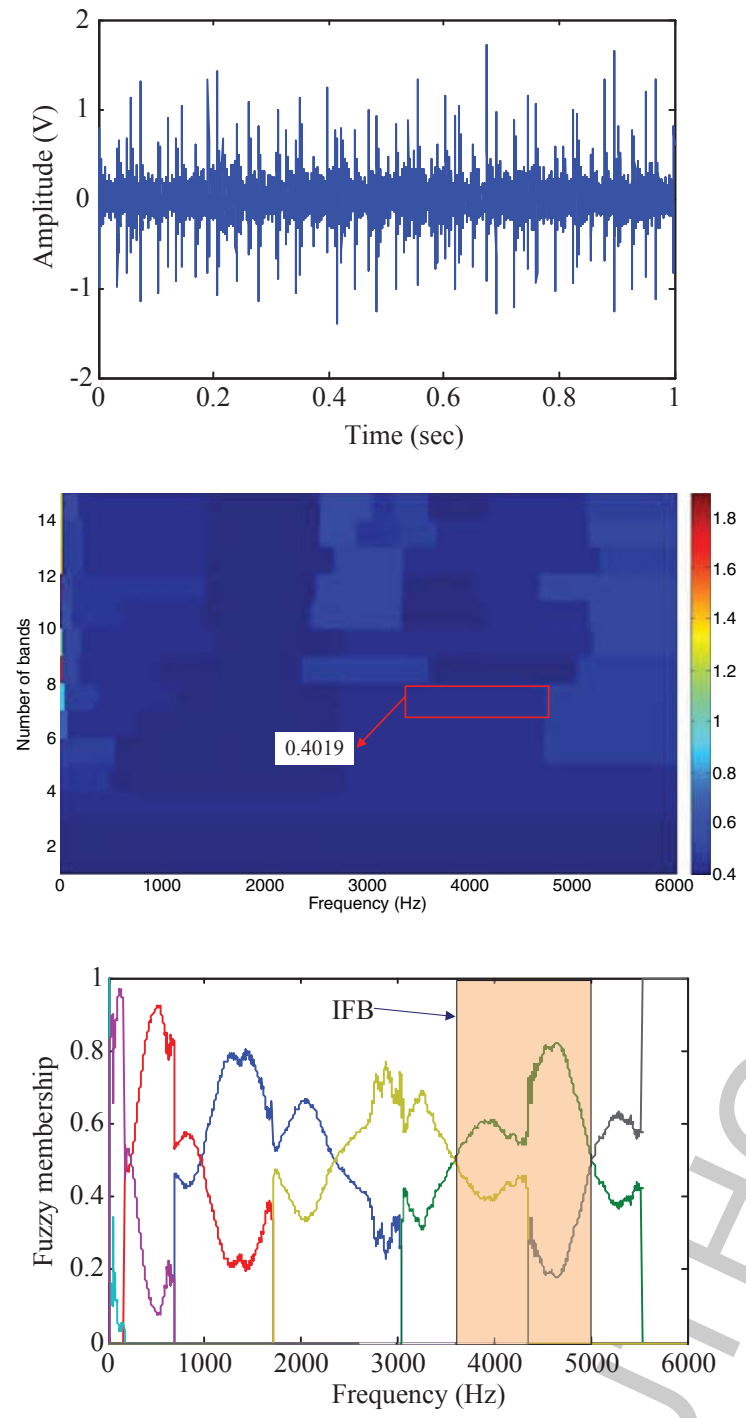

(e) (c)

(a)

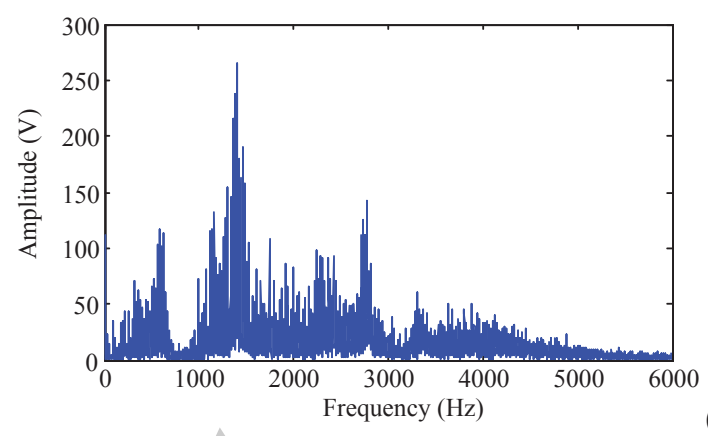

(b)

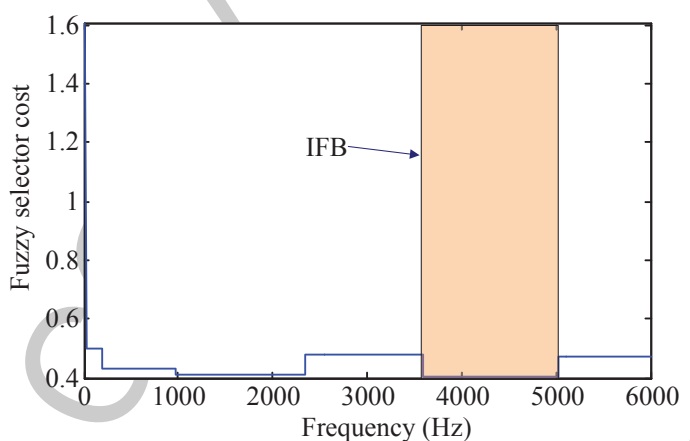

(d)

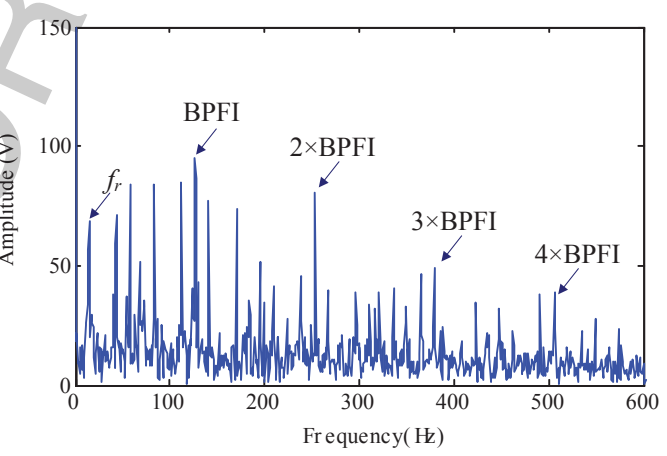

Fig. 6. Experimental result for the bearing with the inner race fault: (a) waveform of the vibratory signal; (b) spectral representation; (c) fuzzy clustering results with $c=1,2, \ldots, 15$; (d) fuzzy comprehensive cost curves when $c_{\mathrm{opt}}=7$; (e) membership curves when $c_{\mathrm{opt}}=7$; and (f) demodulated envelope spectrum from the IFB.

\section{Experimental data analysis}

In this section, an experimental setup as shown in Fig. 5 was built to collect bearing condition data to test the performance of the present approach. As shown in Fig. 5, the output end of a motor (SIEMENS, 3phase, 2.0 HP) was connected to a shaft $(\phi 30 \mathrm{~mm})$ via a coupling. Two flywheels were installed on the shaft as the load, and two rolling element bearings (namely, bearing 1 and bearing 2, SKF 1207 EKTN9/C3) were used to support the shaft at its two ends. To adjust the speed of the motor, an inverter (DANFOSS VLT
$1.5 \mathrm{~kW}$ ) was installed in a control panel. The rotating speed of the motor was monitored by a tachometer (COMPACT VLS5/T/LSR optical sensor) through a data acquisition box (ERBESSD, DAQ 2 in short). To monitor the health condition of the bearing, an accelerometer (PCB ICP 353C03) was mounted on the housing (SKF SNL 507-606) of bearing 1 to collect vibration signals which were fed to another data acquisition box (NI cDAQ-9234, DAQ 1 in short). Both DAQ 1 and DAQ 2 were connected by a laptop (HP Pavilion g4-2055la) for bearing fault detection from the collected signals. During the experiments, 


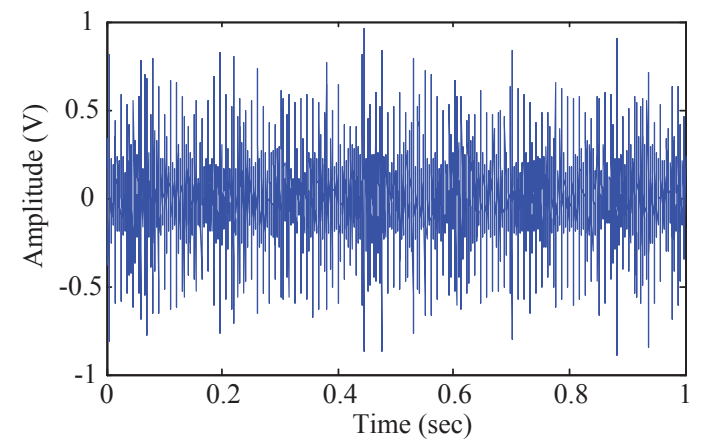

(a)

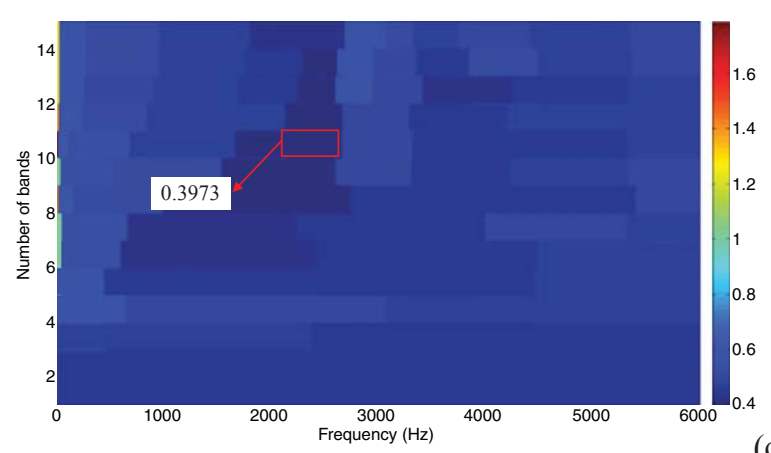

(c)

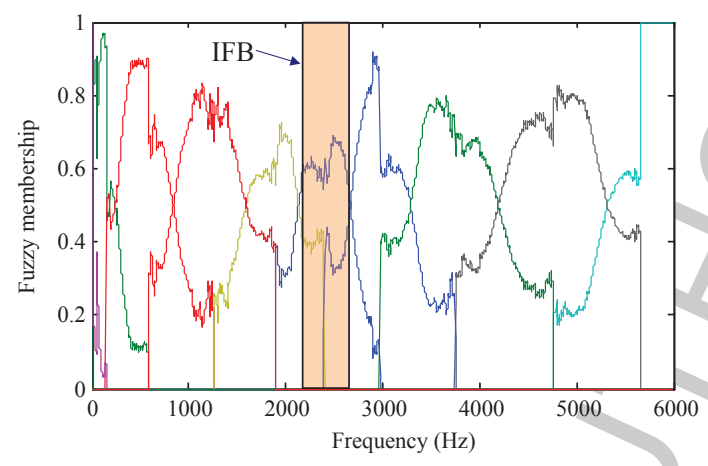

(e)

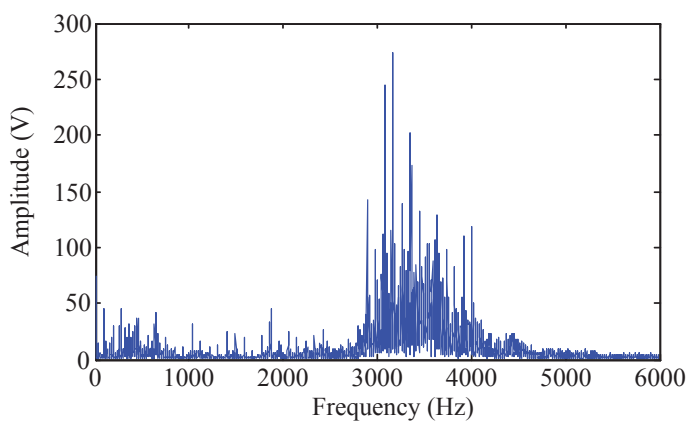

(b)

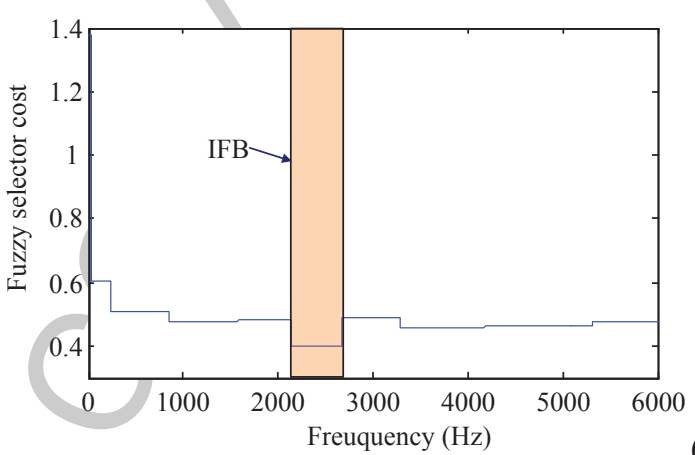

(d)

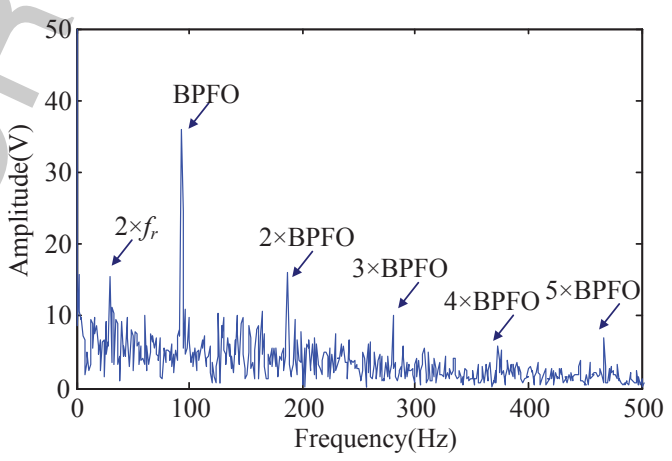

Fig. 7. Experimental result for the bearing with the outer race fault: (a) vibratory signal; (b) Fourier spectrum; (c) clustering results with $c=1,2, \ldots, 15$; (d) fuzzy comprehensive cost curves for $c_{\mathrm{opt}}=10$; (e) membership curves for $c_{\mathrm{opt}}=10$; and (f) demodulated envelope spectrum from the IFB.

three bearings were used to justify the fault detection capability. The first bearing had an inner race fault, the second an outer race fault, and the third no fault. The inner race fault $(\phi 0.9 \mathrm{~mm})$ and the outer race fault $(\phi 1 \mathrm{~mm})$ were pre-planted on the SKF 1207 EKTN9/C3 bearing as displayed also in Fig. 5. The parameters of the bearing to be diagnosed were as follows: diameter of the rolling element $d=8.70 \mathrm{~mm}$, pitch diameter $D=53.5 \mathrm{~mm}$, number of balls (for each row) $n=15$, and contact angle $\alpha=0$. The characteristic frequencies for different faults can be calculated from Equation (27) as: $\operatorname{BPFO}\left(f_{c}\right)=$ $6.3 f r$, and BPFI $\left(f_{c}\right)=8.7 f r$. For the experiments in this section, the sampling frequency was set at $12 \mathrm{kHz}$.

\subsection{Experiment to detect an inner race fault}

In this experiment, the bearing with the inner race fault (right bearing in Fig. 5) was employed as bearing 1. The rotation speed of the shaft was set at $15 \mathrm{~Hz}$ (in the frequency inverter) but the real $f_{r}$ was $14.6 \mathrm{~Hz}$ (measured by the tachometer). The temporal waveform and its Fourier representation of the vibration signal are shown in Fig. 6(a) and (b), respectively. Using the present fuzzy technique, clustering results 

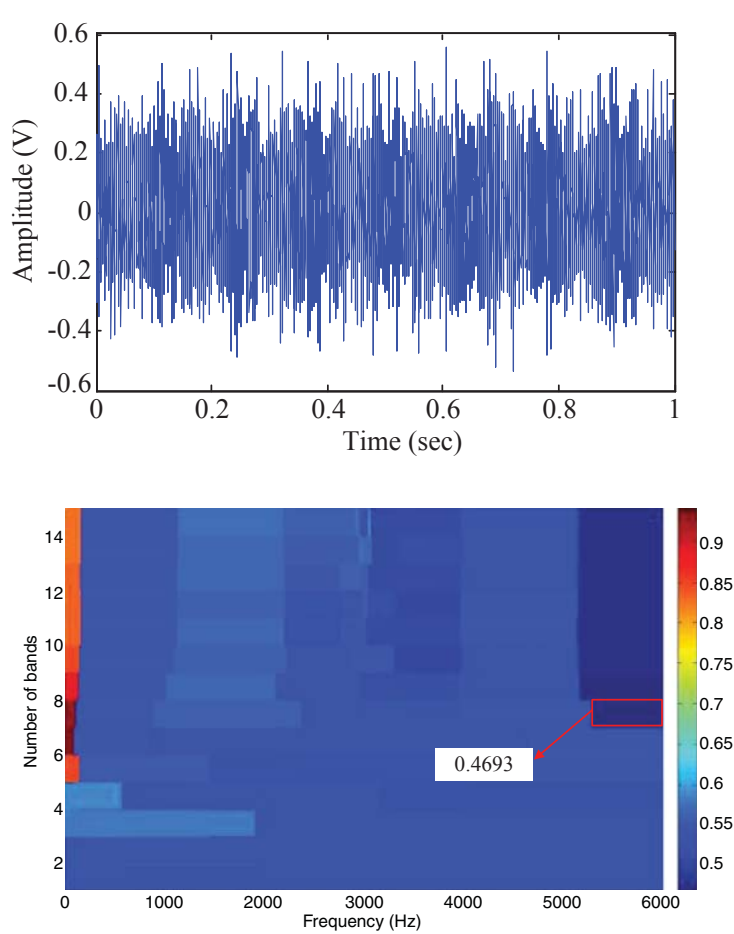

(c)

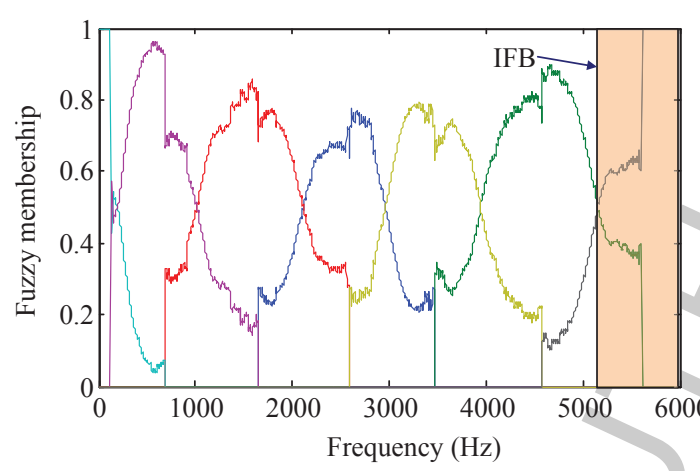

(a)

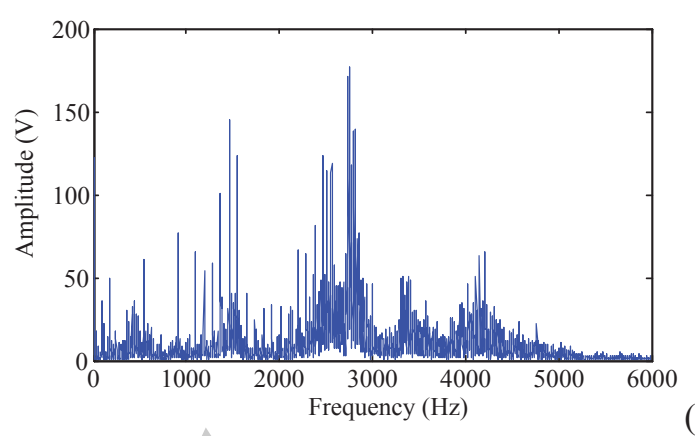

(b)

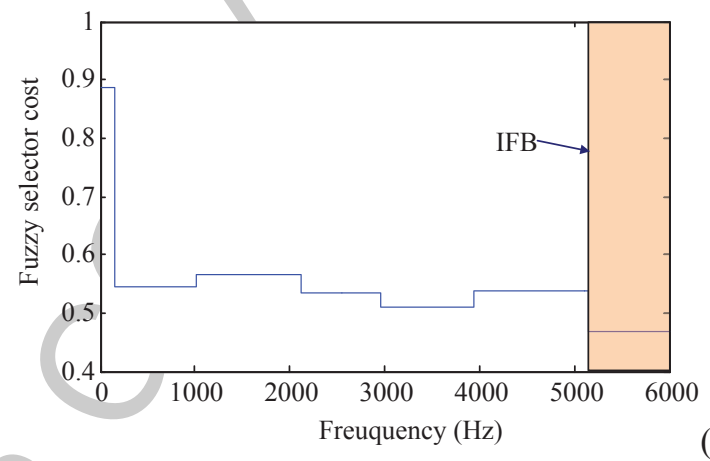

(d)

Fig. 8. Fault detection experiment for the normal bearing: (a) raw signal; and (b) spectral representation; (c) fuzzy clustering results with $c=1,2, \ldots, 15$; (d) fuzzy comprehensive cost curves for $c_{\mathrm{opt}}=7$; (e) membership curves for $c_{\mathrm{opt}}=7$; and (f) fault detection result.

with $c=1,2, \ldots, 15$ are displayed in Fig. 6(c). The smallest fuzzy comprehensive cost was found at $c_{\mathrm{opt}}=7$, for which the fuzzy comprehensive cost curves are highlighted in Fig. 6(d). In this figure, the global minimum cost of 0.4019 is found at [3596, $5018] \mathrm{Hz}$; this is identified as the IFB. To further illustrate the clustering process, the membership curves for $c_{\mathrm{opt}}=7$ are displayed in Fig. 6(e). In this figure, it is evident that the whole frequency spectrum is clustered as 7 segments (i.e., $[1,16] \mathrm{Hz},[17,163]$ $\mathrm{Hz},[164,967] \mathrm{Hz},[968,2342] \mathrm{Hz},[2343,3595]$ $\mathrm{Hz},[3596,5018] \mathrm{Hz}$, and $[5019,6000] \mathrm{Hz})$, among which the 6th segment $([3596,5018] \mathrm{Hz})$ is the IFB.
The IFB was used to produce the optimal demodulated envelope spectrum as displayed in Fig. 6(f). From this figure, one can see up to 4 harmonics of the characteristic frequency BPFI $\left(f_{c}\right)$. This indicates that an inner race fault can be detected for the bearing.

\subsection{Experiment to detect the outer race fault}

The bearing with an inner race fault was replaced by a rolling element bearing with an outer race fault (left bearing in Fig. 5. The rotating speed in this experiment was set at $f_{r}=15 \mathrm{~Hz}$ (the measured rotating frequency was $14.8 \mathrm{~Hz}$ ). The vibratory signal and its 
spectrum are shown in Fig. 7(a) and (b), respectively. The present fuzzy technique was again applied for spectral band clustering. The clustering results with $c=1,2, \ldots, 15$ are displayed in Fig. 7(c), where the smallest fuzzy comprehensive cost can be found at $c_{\text {opt }}=10$. For this optimal clustering $\left(c_{\mathrm{opt}}=10\right)$, the fuzzy comprehensive cost curves and the membership curves are plotted in Fig. 7(d) and (e), respectively. One can observed from Fig. 7(d) that the minimum cost of 0.3973 is related to the IFB [2135, 2668] $\mathrm{Hz}$, which is the same for Fig. 7(e). Through envelope demodulation using the IFB, Fig. 7(f) plots the spectrum $V(f)$ of the optimal demodulated envelope. From this figure, one can identify the BPFO $\left(f_{c}=93.2 \mathrm{~Hz}\right)$ and its second, third, fourth, and fifth harmonics. In contrast, only the second harmonic of the rotation frequency $(14.8 \mathrm{~Hz})$ can be observed due to the filtering effect of the IFB. This reveals that bearing 1 has an outer race defect.

\subsection{Normal bearing diagnosis experiment}

In this subsection, the defective bearing was replaced by a normal one to carry out the fault detection experiment. The vibration signal collected from this configuration is displayed in Fig. 8(a). Figure 8(b) shows the spectral representation of the vibratory measurement. By applying the addressed fuzzy technique for the frequency spectrum, Fig. 8(c) shows the fuzzy clustering results using $c=1,2, \ldots, 15$. It is found that the minimum fuzzy selector cost of 0.4693 is related to $c_{\mathrm{opt}}=7$. To further illustrate the clustering process, for $c_{\text {opt }}=7$, Fig. 8(d) and (e) plot its fuzzy cost and the membership curves, respectively. Using the identified IFB ([2011, 2889] Hz), Fig. 8(f) displays the optimal demodulated envelope spectrum. In this figure, one can observe the rotating frequency and its harmonics. Fortunately, none of the faulty characteristic frequency can be found in the optimal demodulated envelope spectrum. This means that the monitored bearing is in a healthy condition.

It should be noted that the minimum fuzzy comprehensive cost as shown in Fig. 8 (0.4693) is larger than those shown in Fig. 6 (0.4019) and Fig. 7 (0.3973). This is due to the faulty feature of the rolling element bearing. In the fault detection experiment as illustrated in Fig. 8, the bearing is normal so that the clustering cost will be greater than those associated with the faulty cases. Hence, it seems that the minimum fuzzy comprehensive cost is an auxiliary tool for the detection of possible bearing faults.

\section{Conclusions}

In this paper, a fuzzy technique has been reported to determine the informative frequency band (IFB) for bearing fault detection. The frequency spectrum of the bearing vibratory signal was segmented by clusters found by a proposed application specific variant of fuzzy C-means (FCM) clustering. This was capable of generating more meaningful sub-bands than the classical mean partitions. As the original FCM performs poorly in frequency spectrum clustering, the work proposed a new dissimilarity measure based on the selector cost and homogeneity. To better characterize the clustering cost for different selectors, the fuzzy comprehensive evaluation model was applied to combine three commonly used selectors, i.e., kurtosis, smoothness index, and crest factor. With the present fuzzy technique, the IFB can be determined more meaningfully and accurately. The optimal demodulated envelope spectrum filtered by the IFB was then used to detect the existence of a bearing fault. The main contributions of the present technique include: (1) fuzzy comprehensive evaluation by combining different selectors makes the selector cost more effective in determining the IFB; (2) fuzzy clustering as a "soft" segmentation method is more meaningful and finer than classical "hard" mean partitions of the frequency spectrum; and (3) joint fuzzy clustering and fuzzy comprehensive evaluation are capable of determining the optimal IFB to enhance bearing fault detection.

As reported in this paper, only the Fourier transform was introduced for frequency band selection. In addition to the Fourier transform, other timefrequency tools such as wavelet transforms were used for optimal filtering of the bearing vibratory signal. It is noted that the present fuzzy technique can also be used in these cases. The present fuzzy technique using time-frequency tools and their application to rotating machinery fault diagnosis will be investigated in our further work.

\section{Acknowledgments}

This work is supported in part by the Prometeo Project of the Secretariat for Higher Education, Science, Technology and Innovation of the Republic of Ecuador, the National Natural Science Foundation of China (51375517), the Project of Chongqing Science \& Technology Commission (cstc2015jcyjA70007, 
cstc2015jcyjA90003), and the open grant of the CTBU (1456023). The valuable comments and suggestions from the three anonymous reviewers are very much appreciated.

\section{References}

[1] F. Cong, W. Zhong, S. Tong, N. Tang and J. Chen, Research of singular value decomposition based on slip matrix for rolling bearing fault diagnosis, Journal of Sound and Vibration 344 (2015), 447-463.

[2] L. Saidi, J.B. Ali and F. Fnaiech, Application of higher order spectral features and support vector machines for bearing faults classification, ISA Transactions 54 (2015), 193-206.

[3] C. Li and M. Liang, Separation of vibration-induced signal of oil debris sensor for vibration monitoring, Smart Materials \& Structures 20(3) (2011), 045019.

[4] V. Arumugam, A.A.P. Sidharth and C. Santulli, Failure modes characterization of impacted carbon fibre reinforced plastics laminates under compression loading using acoustic emission, Journal of Composite Materials 48(28) (2014), 3457-3468.

[5] R.B. Randall and J. Antoni, Rolling element bearing diagnostics - A tutorial, Mechanical Systems and Signal Processing 25(2) (2011), 485-520.

[6] D. Stefanoiu, Fault diagnosis by fuzzy-statistical reasoning, Journal of Intelligent \& Fuzzy Systems 15(3-4) (2004), 181-194.

[7] D. Wang and Q. Miao, Smoothness index-guided Bayesian inference for determining joint posterior probability distributions of anti-symmetric real Laplace wavelet parameters for identification of different bearing faults, Journal of Sound and Vibration 345 (2015), 250-266.

[8] A. Ghasemloonia and S.E.Z. Khadem, Gear tooth failure detection by the resonance demodulation technique and the instantaneous power spectrum method - a comparative study, Shock and Vibration 18(3) (2011), 503-523.

[9] J. Obuchowski, A. Wyłomańsk and R. Zimroz, Selection of informative frequency band in local damage detection in rotating machinery, Mechanical Systems and Signal Processing 48 (2014), 138-152.

[10] W. Wang, Early detection of gear tooth cracking using the resonance demodulation technique, Mechanical Systems and Signal Processing 15(5) (2001), 887-903.

[11] J. Antoni and R. Randall, The spectral kurtosis: Application to the vibratory surveillance and diagnostics of rotating machines, Mechanical Systems and Signal Processing 20(2) (2006), 308-331.

[12] I. S. Bozchalooi and M. Liang, A smoothness index-guided approach to wavelet parameter selection in signal de-noising and fault detection, Journal of Sound and Vibration 308(1-2) (2007), 246-267.

[13] K.C. Gryllias and I. Antoniadis, A peak energy criterion (P.E.) for the selection of resonance bands in complex shifted Morlet wavelet (CSMW) based demodulation of defective rolling element bearing vibration response, International Journal of Wavelets Multiresolution and Information Processing 7(4) (2009), 387-410.

[14] C. Li, M. Liang and T. Wang, Criterion fusion for spectral segmentation and its application to optimal demodulation of bearing vibration signals, Mechanical Systems and Signal Processing 64-65 (2015), 132-148.
[15] J. Antoni, The infogram: Entropic evidence of the signature of repetitive transients, Mechanical Systems and Signal Processing 74 (2016), 73-94.

[16] T. Barszcz and A. Jabłoński, A novel method for the optimal band selection for vibration signal demodulation and comparison with the Kurtogram, Mechanical Systems and Signal Processing 25(1) (2011), 431-451.

[17] W. He, Z.N. Jiang and K. Feng, Bearing fault detection based on optimal wavelet filter and sparse code shrinkage, Measurement 42(7) (2009), 1092-1102.

[18] C. Li and M. Liang, Time-frequency signal analysis for gearbox fault diagnosis using a generalized synchrosqueezing transform, Mechanical Systems and Signal Processing 26(1) (2012), 205-217.

[19] C. Li, V. Sanchez, G. Zurita, M.C. Lozada and D. Cabrera, Rolling element bearing defect detection using the generalized synchrosqueezing transform guided by time-frequency ridge enhancement, ISA Transactions 60 (2016), 274-284.

[20] P. Flandrin, G. Rilling and P. Goncalves, Empirical mode decomposition as a filter bank, IEEE Signal Processing Letter 11(2) (2004), 112-114.

[21] C. Li, M. Liang, Y. Zhang and S. Hou, Multi-scale autocorrelation via morphological wavelet slices for rolling element bearing fault diagnosis, Mechanical Systems and Signal Processing 31(8) (2012), 428-446.

[22] C. Li and M. Liang, Continuous-scale mathematical morphology-based optimal scale band demodulation of impulsive feature for bearing defect diagnosis, Journal of Sound and Vibration 331 (2012), 5864-5879.

[23] S. Hou, M. Liang, Y. Zhang and C. Li, Vibration signal demodulation and bearing fault detection: A clusteringbased segmentation method, IMeche Proceedings Part C: Journal of Mechanical Engineering Science 228(11) (2014), $1888-1899$.

[24] Y. Wang, X. Ma, M. Xu, Y. Wang and Y. Liu, Vehicle routing problem based on a fuzzy customer clustering approach for logistics network optimization, Journal of Intelligent \& Fuzzy Systems (2015). doi: 10.3233/IFS-151578

[25] W. Niu and H. Zhang, A preference-based recommendation method with fuzzy comprehensive evaluation, Intelligent Decision Technologies 8(3) (2014), 179-187.

[26] P. Su, C. Shang and Q. Shen, A hierarchical fuzzy cluster ensemble approach and its application to big data clustering, Journal of Intelligent \& Fuzzy Systems 28(6) (2015), 2409-2421.

[27] J. Valente de Oliveira and W. Pedrycz, Advances in fuzzy clustering and its applications, John Wiley \& Sons Inc., New York, NY, USA, 2007.

[28] J. Abonyi, B. Feil, S. Nemeth and P. Arva, Modified Gath-Geva clustering for fuzzy segmentation of multivariate time-series, Fuzzy Sets and System 149 (2005), 39-56.

[29] P. Fazendeiro and J. Valente de Oliveira, Observer biased fuzzy clustering, IEEE Transactions on Fuzzy Systems 23(1) (2015), 85-97.

[30] J. Guo, D. Fan, H. Che, Y. Duan, H. Wang and D. Zhang, An approach to network security evaluation of computer network information system with triangular fuzzy information, Journal of Intelligent \& Fuzzy Systems 28(5) (2015), 2029-2035.

[31] C. Li, R. Sanchez, G. Zurita, M. Cerrada, D. Cabrera and R.E. Vásquez, Multimodal deep support vector classification with homologous features and its application to gearbox fault diagnosis, Neurocomputing 168 (2015), 119-127. 